\title{
Beneficial effects of verbalization and visual distinctiveness on remembering and knowing faces
}

\author{
CHARITY BROWN \\ University of Leeds, Leeds, England \\ and \\ TOBY J. LLOYD-JONES \\ University of Kent, Canterbury, England
}

\begin{abstract}
We examined the effect of verbally describing faces upon visual memory. In particular, we examined the locus of the facilitative effects of verbalization by manipulating the visual distinctiveness of the to-be-remembered faces and using the remember/know procedure as a measure of recognition performance (i.e., remember vs. know judgments). Participants were exposed to distinctive faces intermixed with typical faces and described (or not, in the control condition) each face following its presentation. Subsequently, the participants discriminated the original faces from distinctive and typical distractors in a yes/no recognition decision and made remember/know judgments. Distinctive faces elicited better discrimination performance than did typical faces. Furthermore, for both typical and distinctive faces, better discrimination performance was obtained in the description than in the control condition. Finally, these effects were evident for both recollection- and familiarity-based recognition decisions. We argue that verbalization and visual distinctiveness independently benefit face recognition, and we discuss these findings in terms of the nature of verbalization and the role of recollective and familiaritybased processes in recognition.
\end{abstract}

Although our capacity for face recognition is typically good (see, e.g., Shapiro \& Penrod, 1986), descriptions of faces are often vague and imprecise (Ellis, Shepherd, \& Davies, 1980; Lindsay, Martin, \& Webber, 1994). The disparity between these abilities suggests that remembering faces is essentially a nonverbal activity. Yet studies have shown that describing a face can influence subsequent recognition. Whereas a number of studies have shown facilitative effects of the verbalizing of faces (e.g., Bloom \& Mudd, 1991; Bower \& Karlin, 1974; Mueller, Courtois, $\&$ Bailis, 1981), in others verbal interference has been observed (see Schooler, 2002, for a recent review). Clear accounts of these phenomena remain elusive, however. The focus of the present article is verbal facilitation. Using a novel paradigm, we examined the locus of the facilitative effects of verbalization on face memory and, in particular, whether the benefit of verbalization is mediated by the visual representation of the face in memory.

Research on verbal facilitation has focused on the levelsof-processing effect (see Craik \& Lockhart, 1972). Sev-

This research was carried out while C.B. was a postdoctoral researcher at the University of Kent. This research was supported by ESRC Postdoctoral Fellowship T026-27-1240 to C.B. and by ESRC Research Grant RES000-23-0057 to T.J.L.-J. Correspondence concerning this article should be addressed to C. Brown, Institute of Psychological Sciences, University of Leeds, Leeds LS2 9JT, England (e-mail: psccbr@leeds.ac.uk). eral studies have shown that making trait judgments (e.g., niceness) to unfamiliar faces at encoding leads to better recognition than does making physical judgments, such as those of gender (see, e.g., Bower \& Karlin, 1974; Mueller et al., 1981; Patterson \& Baddeley, 1977). Accounts of the levels-of-processing effect can be broadly separated into those that emphasize either visual or semantic processing. Visual-processing accounts argue that facilitation arises as a function of the quantity or quality of visual information encoded about the face. Facilitation may be due to a greater number of features being attended to and stored during encoding (the feature quantity account; e.g., Blaney \& Winograd, 1978; Courtois \& Mueller, 1979; Winograd, 1978, $1981)$ or to the encoding of more holistic impressions of the face (i.e., the interrelations between features), in addition to feature-based information (the holistic account; e.g., Berman \& Cutler, 1998; Wells \& Hryciw, 1984). Alternatively, semantic-processing accounts suggest that facilitation may be due to the addition of semantic associations to the described face, which benefit retrieval (e.g., Anderson \& Reder, 1979; Bruce \& Young, 1986; Ryan \& Schooler, 1994, cited in Schooler, Ryan, \& Reder, 1996).

Attempts to distinguish between visual- and semanticprocessing accounts have provided mixed conclusions. For instance, Bloom and Mudd (1991) have presented evidence that is consistent with a visual-processing, rather than a semantic-processing, account. They observed that as processing depth increases (i.e., from gender to per- 
sonality judgments), so the number of participants' eye movements, the time spent inspecting the face, and subsequent recognition performance increase. Moreover, when they held the number of eye movements and inspection time constant, recognition performance did not increase with processing depth. These findings are consistent with a visual-processing account that suggests that as depth of processing increases, more facial features are encoded, which enhances the visual representation of the face in memory and benefits recognition.

In contrast, Klatzky, Martin, and Kane (1982), for instance, reported findings supporting a semantic-processing account. They found that faces presented in congruent semantic contexts (i.e., a face previously rated as stereotypical of a particular occupational category was labeled as belonging to that same category) were better recognized than were faces in incongruent contexts. However, at the same time, new faces rated as belonging to the same stereotypical category as that of previously studied faces were more often falsely recognized (i.e., responded to as old) than were new faces that were not. This suggests that faces can be associated with semantic information that augments the visual representation of the face. However, when the semantic information is sufficiently abstract, it may bias responses to new faces that are similar to the category stereotype. Thus, semantic processing may also influence the visual encoding of faces that have not been encountered previously (see also Kerr \& Winograd, 1982).

Finally, this picture is complicated by the fact that there are a number of studies that have established interfering effects of verbalizing a face upon subsequent recognition, a phenomenon termed verbal overshadowing (e.g., Meissner, Brigham, \& Kelley, 2001; Schooler \& EngstlerSchooler, 1990; see Schooler, 2002, for a review). Recently, Meissner et al. (2001) proposed a misinformation account of verbal interference whereby nonveridical information elicited by a description impacts unfavorably upon the visual memory for the target face (see also Meissner \& Brigham, 2001). However, there are situations in which verbal interference has been found to extend beyond the described stimulus (Brown \& Lloyd-Jones, 2002, 2003; Dodson, Johnson, \& Schooler, 1997; Westerman \& Larsen, 1997). This, and other evidence, is consistent with a transfer-inappropriate-processing account, which suggests a more generalized form of interference (e.g., Schooler, 2002; Schooler, Fiore, \& Brandimonte, 1997). In essence, following encoding, the application of verbal processes interferes in some general way with the utilization of nonverbal processes, which are useful for recognition.

\section{A New Paradigm: Verbal Facilitation Versus Interference}

We present a novel paradigm, in which we aim to establish verbal facilitation across a relatively large number of faces. The general paradigm is as follows. In a study phase, participants view and then describe (or not, in the control condition) each of 24 faces. Subsequently, they discriminate the original 24 (old) faces from 24 (new) dis- tractors in a yes/no recognition task. Two previous studies, which have shown both beneficial and interfering effects of verbalization across multiple faces, are of particular relevance here.

Exposure to multiple faces can lead to both proactive (i.e., encoding of faces is impaired by prior exposure to earlier faces) and retroactive (i.e., the retrieval of faces is impaired by prior exposure to earlier recognition tests) interference (e.g., Davies, Shepherd, \& Ellis, 1979; Deffenbacher, Carr, \& Leu, 1981). However, Ryan and Schooler (1994, cited in Schooler et al., 1996) found evidence that verbalization may act to reduce this interference. In their study, participants viewed and described (or not, in the control condition) four target faces. They were then given four recognition tests, presented in an order corresponding to that in which the faces had been originally encountered. For no-description participants, recognition performance declined substantially over the four recognition tests, presumably with the buildup of interference. However, for description participants, substantially less interference was observed. This suggests that verbalizing individual faces may protect them from the interference that arises with exposure to other faces within a similar encoding context.

Nevertheless, Brown and Lloyd-Jones (2003) have found interfering effects of verbalization on face recognition in a paradigm similar to that presented here. Participants viewed 12 to-be-remembered stimuli (faces or cars) and then described (or not, in the control condition) a related stimulus (i.e., another face or car). Verbal interference arose for both face and car recognition. They argued that verbalization encourages a shift toward featural processing, at the expense of holistic processing, which is generally more beneficial for recognition of stimuli that are highly visually similar. In the present paradigm, therefore, verbal interference, rather than facilitation, is possible. However, the participants here were asked to describe each face in the series, rather than a single face following a series of faces, and this is likely to be an important factor in determining the facilitative effects of verbalization (Brown \& Lloyd-Jones, 2002).

We will turn now to the issue of the locus of the effects of verbal facilitation, which we examined by manipulating the visual distinctiveness of the face and measuring performance through use of the remember/know procedure (Tulving, 1985).

\section{Verbalization and Visual Distinctiveness}

It is a robust finding that visually distinctive faces are recognized more accurately and more quickly than typical faces (e.g., Light, Kayra-Stuart, \& Hollander, 1979; Valentine \& Bruce, 1986). Indeed, this finding has been used to constrain a number of models of face memory (e.g., Lewis, 2004; Valentine, 1991; Valentine \& Endo, 1992). One such influential model is the exemplar-based face space model developed by Valentine (1991; see also Lewis, 2004, for a revised exemplar-based model). Here, faces are represented on a set of shared dimensions within multidimensional face space. Their positioning reflects 
interitem similarity, with more similar (i.e., typical) faces located in closer proximity than less similar (i.e., distinctive) faces. In this way, typical faces are more difficult to learn, due to the fact that many nearby similar faces could interfere with recognition.

Face space models do not specify the dimensions by which faces are represented in memory (Lewis, 2004). However, researchers often define these dimensions as visual aspects of the face (Benson \& Perrett, 1994; Bruce, Burton, \& Dench, 1994). For example, Leder and Bruce (1998) found that both local (i.e., a single feature) and relational (e.g., the relationship between two features) visual information contributes to face distinctiveness. Moreover, some view the dimensions as reflecting statistical properties of visual-based variation among faces (e.g., Burton, Bruce, \& Hancock, 1999). In accord with this notion, a large and reliable correlation exists between face distinctiveness and measures of visual-based variation (i.e., eigenfaces derived from principle components analysis; Hancock, Burton, \& Bruce, 1996; O'Toole, Deffenbacher, Valentin, \& Abdi, 1994).

The evidence outlined above suggests that the effects of face distinctiveness are likely mediated by the visual representation stored in memory. If verbalization also impacts upon this visual representation, interactive effects of verbalization and face distinctiveness on recognition memory may be apparent: Verbalization may act to enhance the visual representation of a face, allowing typical faces to be better distinguished from similar faces in memory. Conversely, verbal facilitation may be less apparent for distinctive faces, which already possess visually distinct representations.

\section{Remembering and Knowing}

Models of recognition memory may be divided broadly into one-process and two-process accounts (see, e.g., Rugg \& Yonelinas, 2003, and Yonelinas, 2002, for recent reviews). Single-process models assume that recognition judgments are based on the target item's familiarity or its total similarity to the contents of memory. Dual-process accounts propose that a second, slower, recall-like process operates as well, which is more accurate for fine discriminations between items. Familiarity is thought to reflect a purely quantitative strength-like memory signal, whereas recollection yields qualitative information about the previous study events (such as when or where an item was studied). One method of measuring recollection and familiarity is the remember/know procedure (Tulving, 1985). This requires participants to introspect about the basis of their recognition memory judgments and report whether they recognize items on the basis of remembering qualitative information about the study event (remember responses) or on the basis of knowing the item is familiar in the absence of recollection (know responses).

The effect of verbalization on recollection and familiarity has not been studied previously. Nevertheless, verbalization may benefit recollection more than it does familiarity. For instance, recollection benefits more from elaborative or deeper encoding (see Yonelinas, 2002, for a comprehensive review) than does familiarity. Furthermore, visual distinctiveness may also benefit recollection more than it does familiarity. For instance, Mäntylä (1997) found that focusing on differences between faces (by rating facial distinctiveness) led to better recognition performance than did focusing on similarities among faces (by categorizing faces into student-type categories, such as sporty or intellectual). Importantly, this benefit was limited to remember responses to items that evoked some specific recollection from the study phase (see also Brandt, Macrae, Schloerscheidt, \& Milne, 2003, for a comparable finding).

In the following experiment, we examined the effects of verbalization and visual distinctiveness on face recognition, using the remember/know procedure as a measure of recognition performance.

\section{METHOD}

\section{Participants}

One hundred sixty-two participants (135 females and 27 males) from the University of Kent participated in partial fulfillment of a course requirement. All were native English speakers, with normal or corrected-to-normal vision. Eighty-four were assigned to the description condition, and 78 to the no-description condition.

\section{Materials and Apparatus}

Prior to the experiment, 12 participants from the University of Kent (who did not take part subsequently) were asked to rate a set of faces in terms of their distinctiveness. The stimuli were grayscale head-and-shoulders photographs of 96 Caucasian men. Fifty photographs were taken from the University of Stirling Psychology Department Psychological Image Collection (pics.psych.stir.ac.uk), and 46 photographs from the Computer Vision Laboratory, Faculty of Computer and Information Science, University of Ljubljana, Slovenia (www.lrv.fri.uni-lj.si/facedb.html). None of the faces had any distinctive marks or wore glasses or a beard, and the photographs were edited to remove clothing cues. A $6 \times 8 \mathrm{~cm}$ full frontal image of each face was printed onto paper, and each face was allocated a number. The participants rated each of the 96 faces on a 7-point scale from 1 (typical) to 7 (distinctive), using instructions taken from Valentine and Bruce (1986). Prior to the rating task, the faces were shuffled so that each participant rated the faces in a different order. The participants were also asked to look through all the faces to obtain an idea of the range of faces available to them and to consider the whole 7-point scale when making their decisions. The participants were then provided with a response sheet containing ninety-six 7-point scales. Next to each scale, the participants wrote the number of the face that they were evaluating.

The mean rating across the 12 participants for each face was computed, and a median split was carried out. Faces with a mean rating of 3.87 or above were grouped as distinctive, and those with a mean rating below 3.87 were grouped as typical. The mean ratings for the typical and distinctive face sets were $3.16(S D=0.43)$ and 4.50 $(S D=0.48)$, respectively.

To ensure that the recognition task involved face recognition, rather than image recognition, two views of each face were used (Baddeley \& Woodhead, 1983; Sporer, 1991). For each face, a full frontal view was presented during the study phase, whereas a threequarter view (facing left) was presented at test.

The 96 faces were divided into two stimulus blocks of 48 faces (each including 24 typical and 24 distinctive faces). Within each stimulus block, the faces were randomly divided into two sets of 24 faces (each including 12 typical and 12 distinctive faces), providing four sets of 24 faces in total. The stimuli were presented on stand-alone PCs using E-Prime presentation software. Face stimuli 
appeared in the center of the computer screen, and each stimulus was created within a surface area of $10 \times 8 \mathrm{~cm}$. The participants were run in groups of 20 to 55. All the participants completed the experiment at separate PCs and wore headphones.

\section{Design and Procedure}

A mixed factorial design was employed, with description (description vs. no description) as a between-participants factor and visual distinctiveness (typical vs. distinctive) as a within-participants factor. The dependent variables were taken from signal detection theory and were discrimination $\left(d^{\prime}\right)$, and response bias $(C)$. In addition, remember and know responses were recorded for hits and false alarms. Accuracy in the recognition test was measured by a keypress response.

Each participant viewed faces taken from a single stimulus block (see the Materials and Apparatus section). Each block comprised 24 faces (including 12 typical and 12 distinctive faces) in the study phase and the same 24 faces as targets (i.e., old items) plus 24 distractors (i.e., new items, which consisted of 12 typical and 12 distinctive faces) in the test phase. In this way, four sets of 24 faces were rotated across the description and no-description conditions, so that each set appeared equally often as either targets or distractors for equal numbers of participants (i.e., for 20 participants within each condition).

The following procedure was adopted for both the description and the no-description conditions. In the study phase, the participants viewed 24 sequentially presented faces. Each face remained on the screen for $5 \mathrm{sec}$ and was preceded by a fixation cross presented for $250 \mathrm{msec}$. Faces were presented in a pseudorandom order in such a way that no more than 3 typical or distinctive faces could appear in an unbroken sequence. Following presentation of each face, there was a 15 -sec interval during which the participants wrote a description or completed a filler task (details provided below). At the end of the $15 \mathrm{sec}$, the computer sounded to alert the participants to return their attention to the screen, and the participants pressed the space bar on the keyboard to view the next face. Prior to viewing the faces, the participants were instructed to study each face for the whole time that it appeared on the screen. In addition, the participants were informed that they would later be asked to recognize the faces that they were about to see but that, in the recognition test, the faces would be presented in a view different from that in the study phase.

Prior to the study phase, the participants were provided with instructions concerning the experimental manipulation. In the description condition, the participants were told that in the 15 -sec interval that followed each face, they would have to write a description of the face that they had just viewed. The precise description instructions were as follows: "Please be as complete in your description as possible, so that another person seeing only your description, could get as accurate an idea as possible of what the face is like." In the no-description condition, a number was presented on the computer screen from which the participants had to count backward in intervals of 3, writing down the numbers on a blank sheet of paper as they counted back.

Immediately following the study phase, recognition was tested in a yes/no decision task in which the three-quarter views of the 24 study faces were mixed randomly with three-quarter views of 24 new faces. The participants were instructed to respond yes if the face had appeared in the study phase and no if it had not. The recognition decision was indicated by pressing one of two keys ( $\mathrm{Z}$ or $\mathrm{M})$ on the computer keyboard. Decision key mapping and hand dominance were counterbalanced. Each face remained in view until the participant responded. The participants were instructed to respond as quickly and accurately as possible.

In addition, the participants were asked to further make a remember or know judgment to those faces that they had responded yes to in the recognition test. Following a yes response, the face was replaced by a prompt to press either $\mathrm{R}$ for remember or $\mathrm{K}$ for know on the keyboard. Following a remember or know response (or a no recognition decision), a prompt appeared instructing the participants to rest their fingers on the recognition decision keys ( $\mathrm{Z}$ and $\mathrm{M})$ and to press the space bar to view the next face. Prior to completing the recognition test, the participants were provided with instructions concerning the definitions of remember/know judgments. These definitions were adapted from Dewhurst and Conway (1994) and were as follows:

When you see a face in this test that you recognize, you may be able to remember specific details about seeing the face in the earlier study phase. You may, for example, recollect the thoughts or feelings that the face evoked when you saw it earlier, or some aspect of the face's physical appearance. In short, any detail that supports your belief that the face appeared in the earlier study phase. For other faces, you simply know that they appeared earlier. These faces may feel familiar, but you cannot recall their actual occurrence in the earlier list. Therefore, every time you recognize a face press either $\mathrm{R}$ for remember if you can remember specific details of the face's earlier occurrence, or $\mathrm{K}$ for know if you recognize the face from the earlier study phase but you cannot recollect its actual occurrence.

\section{RESULTS}

Analyses of accuracy were carried out to assess whether verbalization and visual distinctiveness influenced discrimination $\left(d^{\prime}\right)$ or response bias $(C)$. Statistical analyses of discrimination and response bias were calculated according to the prescriptions set out by Snodgrass and Corwin (1988). That is, difficulties arise for the signal detection theory model at hit or false alarm rates of 1 or 0 . Therefore, we transformed accuracy data by adding .5 to each frequency and dividing by $N+1$, where $N$ is the number of old or new trials/stimuli. For $d^{\prime}$, larger values indicate a greater ability to discriminate between old and new items in the recognition test. For $C$, values above 0 indicate a conservative bias (i.e., a tendency to respond no), and values below 0 a liberal bias, in the recognition test. For response bias, there were no significant main effects or interactions, and therefore, the analyses are not reported.

In addition, we examined whether verbalization and visual distinctiveness influenced the participants' recollective experience in face recognition. There is some disagreement concerning the appropriateness of different measurement methods that have been used to dissociate recollection- and familiarity-based recognition. Two contrasting models are the exclusivity (e.g., Gardiner \& Java, 1990; Gardiner \& Parkin, 1990) and the independence (Yonelinas, 2002; Yonelinas \& Jacoby, 1995) models. The exclusivity view assumes that remember and know judgments provide measures of two subjective states of awareness associated with memory performance. Remember judgments reflect conscious recollection, whereas know responses reflect conscious memorial states of familiarity. These two states are assumed to be mutually exclusive and map directly onto the absolute proportions of remember and know responses (e.g., Gardiner, 1988; Parkin, Gardiner, \& Rosser, 1995). Critics, however, argue that the exclusivity approach makes the implicit assumption that the processes underlying recollection- and familiarity-based recognition are also mutually exclusive (Yonelinas, 2002; Yonelinas \& Jacoby, 1995; although see Richardson-Klavehn, 
Gardiner, \& Java, 1996). A contrasting view is that the processes underlying recollection- and familiarity-based recognition are independent (Yonelinas, 2002; Yonelinas \& Jacoby, 1995). In this approach, remember and know responses are used as a basis for estimating the relative contributions of these independent processes to recognition memory. Since participants are instructed to respond remember whenever an item is recollected, the proportion of remember responses can be taken as a relatively unbiased measure of recollection. However, the proportion of know responses will underestimate the probability that a given item is familiar. This is because participants are instructed to give a know response whenever an item is familiar but not recollected, whereas under the independence assumption there will be some proportion of items that are both recollected and familiar. Therefore, estimates of familiarity are derived by dividing the proportions of know responses by one minus the proportions of remember responses (Yonelinas \& Jacoby, 1995).

We do not wish to make strong claims concerning the relationship between remember and know judgments and the underlying processes of recollection and familiarity, respectively. For this reason, we assess knowing as the state of awareness indexed directly by know responses (e.g., Gardiner, 1988; Parkin et al., 1995), and we also use

Table 1

Means and Standard Deviations for Discrimination, Response Bias, Hits, and False Alarms (FAs) as a Function of Description, Distinctiveness, and Remember/Know Judgments

\begin{tabular}{|c|c|c|c|c|}
\hline \multirow[b]{2}{*}{ Measure } & \multicolumn{2}{|c|}{ Typical Faces } & \multicolumn{2}{|c|}{ Distinctive Faces } \\
\hline & $M$ & $S D$ & $M$ & $S D$ \\
\hline \multicolumn{5}{|c|}{ Description Condition } \\
\hline$d^{\prime}$ & 0.82 & 0.52 & 1.20 & 0.59 \\
\hline C & .25 & .36 & .23 & .34 \\
\hline \multicolumn{5}{|c|}{ Remember responses } \\
\hline Hits & .29 & .17 & .37 & .17 \\
\hline FAs & .10 & .10 & .09 & .10 \\
\hline \multicolumn{5}{|l|}{ Know responses } \\
\hline Hits & .27 & .15 & .27 & .15 \\
\hline FAs & .16 & .15 & .11 & .10 \\
\hline \multicolumn{5}{|c|}{ Familiarity estimates } \\
\hline Hits & .37 & .19 & .43 & .22 \\
\hline FAs & .18 & .16 & .13 & .11 \\
\hline \multicolumn{5}{|c|}{ No-Description Condition } \\
\hline$d^{\prime}$ & 0.31 & 0.46 & 0.68 & 0.65 \\
\hline$C$ & .30 & .36 & .24 & .35 \\
\hline \multicolumn{5}{|c|}{ Remember responses } \\
\hline Hits & .20 & .13 & .28 & .15 \\
\hline FAs & .12 & .14 & .11 & .11 \\
\hline \multicolumn{5}{|l|}{ Know responses } \\
\hline Hits & .24 & .14 & .26 & .15 \\
\hline FAs & .20 & .15 & .18 & .14 \\
\hline \multicolumn{5}{|c|}{ Familiarity estimates } \\
\hline Hits & .30 & .16 & .36 & .20 \\
\hline FAs & .23 & .16 & .20 & .16 \\
\hline
\end{tabular}

Note-For $d^{\prime}$, larger values indicate a greater ability to discriminate between old and new items. For $C$, values above 0 indicate a conservative bias, and values below 0 a liberal bias. Familiarity estimates are derived by dividing the proportions of know responses by one minus the proportion of remember responses (Yonelinas \& Jacoby, 1995), whereas remember responses are a direct estimate of recollection. know responses as a basis for estimating the underlying process of familiarity (e.g., Yonelinas, 2002; Yonelinas \& Jacoby, 1995).

Table 1 shows discrimination, response bias, hits, and false alarms as a function of description, visual distinctiveness, and response type (i.e., remember/know). Analyses consisted of two-way mixed design ANOVAs with description (description vs. no description) as a betweenparticipants factor and visual distinctiveness (distinctive vs. typical) as a within-participants factor. Data were analyzed in three ways. First, we calculated $d^{\prime}$ as a measure of accuracy. Second, the proportion of hits and false alarms were analyzed separately for remember and know responses (i.e., the number of old [new] remember or know responses was divided by the total number of old [new] items). Third, we computed separate familiarity estimates for hits and false alarms, as suggested by Yonelinas (2002). Note that the proportion of remember responses already provides a direct estimate of recollection. Following these analyses, we will present post hoc analyses of description quality, in order to examine the nature of the descriptions in detail.

\section{Discrimination}

There was a main effect of description $\left[d^{\prime}, F(1,160)=\right.$ $\left.65.51, M S_{\mathrm{e}}=0.33, p<.001\right]$. The ability of participants to discriminate between old and new faces was better in the description than in the no-description condition. In addition, there was a main effect of distinctiveness $\left[d^{\prime}\right.$, $\left.F(1,160)=37.74, M S_{\mathrm{e}}=0.30, p<.001\right]$. Discrimination performance was better for distinctive than for typical faces [note that there was no description $\times$ distinctiveness interaction; $d^{\prime}, F(1,160)=0.03, M S_{\mathrm{e}}=0.30, p=$ n.s.].

\section{Remember and Know Responses}

For correct remember responses (i.e., hits), there was a main effect of description $\left[F(1,160)=23.32, M S_{\mathrm{e}}=\right.$ $0.03, p<.001]$. More remember responses were made in the description than in the no-description condition. In addition, there was a main effect of distinctiveness $\left[F(1,160)=22.68, M S_{\mathrm{e}}=0.02, p<.001\right]$. Distinctive faces elicited more remember responses than did typical faces. For correct know responses, there was no main effect or interaction.

False alarms were also analyzed. For incorrect remember responses, there were no main effects or interactions. However, for incorrect know responses, there was a main effect of description $\left[F(1,160)=9.27, M S_{\mathrm{e}}=0.02, p<\right.$ $.01]$. There were fewer false know responses in the description than in the no-description condition. In addition, there was a main effect of distinctiveness $[F(1,160)=$ $\left.7.67, M S_{\mathrm{e}}=0.01, p<.01\right]$. Fewer false know responses were made to distinctive than to typical faces.

\section{Familiarity Estimates}

For correct recognition decisions, there was a main effect of description $\left[F(1,160)=8.45, M S_{\mathrm{e}}=0.04, p<\right.$ .01]. Familiarity estimates were higher in the description than in the no-description condition. In addition, there was 
a main effect of distinctiveness $\left[F(1,160)=7.73, M S_{\mathrm{e}}=\right.$ $0.03, p<.01]$. Familiarity estimates were higher for distinctive than for typical faces.

For incorrect recognition decisions, there was a main effect of description $\left[F(1,160)=10.67, M S_{\mathrm{e}}=0.03, p=\right.$ $.001]$. Familiarity estimates were lower in the description than in the no-description condition. In addition, there was a main effect of distinctiveness $\left[F(1,160)=7.68, M S_{\mathrm{e}}=\right.$ $0.01, p<.01]$. Familiarity estimates were lower for distinctive than for typical faces.

\section{DESCRIPTION QUALITY}

To assess post hoc whether the quality of the participants' descriptions was related to facial distinctiveness, two independent judges examined the proportion (i.e., the total number of each descriptor type divided by the total number of descriptors) of featural versus holistic descriptors, as well as the mean total amount of information per face that was generated (see Brown \& Lloyd-Jones, 2002, 2003). Featural descriptors were taken from Brown and Lloyd-Jones (2002) and consisted of judgments about isolated facial features: size or shape of the chin, lips, nose, eyes, eyebrows, and forehead; distance between two features (such as eyes close together); and hair length and texture. Holistic descriptors were classified according to categories derived from Coin and Tiberghien (1997) and consisted of judgments concerning personality (e.g., jolly, studious, intelligent, or pleasant), weight, age, global face structure (e.g., head shape, skin tone, expression, and comparative judgments about features, such as chin was narrower than forehead), and hairstyle (see also O'Toole et al., 1994, who classified hairstyle as a holistic facial aspect). A small number of descriptors that did not fit into either of these two categories were classified as other descriptors. For the 84 participants' descriptions, collapsing across both typical and distinctive faces, the correlations between the two judges were $r=.96$ for featural descriptors, $r=.90$ for holistic descriptors, and $r=.98$ for the mean number of descriptors. The average of the two judges' ratings was taken for each coding category, and the proportions of featural and holistic descriptors were calculated across the 12 typical and 12 distinctive faces, respectively. In addition, we calculated the mean number of descriptors generated for each of the 12 typical and 12 distinctive faces.

Separate $t$ tests examined whether descriptions of distinctive and typical faces differed in terms of the three description quality measures. There was no difference in description quality.

1. There was no difference between typical and distinctive face descriptions in terms of the proportion of featural descriptors generated $[t(83)=-0.39$, n.s.]. The mean proportion of featural descriptors was $.66(S D=0.13)$ for typical face descriptions and $.66(S D=0.12)$ for distinctive face descriptions.

2. There was no difference between typical and distinctive face descriptions in terms of the proportion of holistic descriptors generated $[t(83)=0.31$, n.s. $]$. The mean proportion of holistic descriptors was $.33(S D=0.13)$ for typical face descriptions, and .34 $(S D=0.12)$ for distinctive face descriptions.

3. There was no difference between typical and distinctive face descriptions in terms of the mean number of descriptors generated $[t(83)=1.13$, n.s. $]$. The mean number of descriptors was $3.72(S D=0.67)$ for typical face descriptions and $3.76(S D=0.69)$ for distinctive face descriptions.

We also examined whether there was a correlation between the participants' description quality and recognition performance. Tables 2 and 3 show the correlations between measures of description quality, discrimination, correct remember/know responses, and familiarity estimates for typical and distinctive faces, respectively.

For typical faces, a decrease in the proportion of featural descriptors and an increase in the proportion of holistic descriptors was associated with an increase in correct remember responses. In contrast, an increase in the proportion of featural descriptors and a decrease in the proportion of holistic descriptors was associated with an increase in correct know responses. (Note that these contrasting effects arise because both featural and holistic descriptors, and remember and know hits, are not independent; if remember responses are positively correlated with holistic

Table 2

Correlations Between Description Quality and Accuracy Measures for Typical Faces

\begin{tabular}{lccccccc}
\hline \multicolumn{1}{c}{ Variables } & Featural & Holistic & Total & $\begin{array}{c}\text { Description } \\
d^{\prime}\end{array}$ & $R$ (hits) & $K$ (hits) & $\begin{array}{c}\text { Familiarity } \\
\text { Estimate }\end{array}$ \\
\hline Featural & - & $-.99^{* *}$ & -.16 & -.10 & $-.35^{* *}$ & $.32^{* *}$ & .19 \\
Holistic & & - & .18 & .11 & $.35^{* *}$ & $-.32^{* *}$ & -.19 \\
Total & & & - & .07 & .06 & .004 & .01 \\
Description $d^{\prime}$ & & & & - & $.34^{* *}$ & .12 & $.28^{* *}$ \\
$R$ (hits) & & & & & - & $-.52^{* *}$ & -.16 \\
$K$ (hits) & & & & & & - & $.90^{* *}$ \\
Familiarity estimate & & & & & & & -
\end{tabular}

Note-Featural, proportion of featural descriptors (featural/featural + holistic + other); Holistic, proportion of holistic descriptors (holistic/featural + holistic + other); Total, mean number of descriptors (holistic + featural + other; derived from each of the 12 target faces); Description $d^{\prime}, d^{\prime}$ scores in the description condition; $R$ (hits), correct remember responses to old faces; $K$ (hits), correct know responses to old faces; Familiarity Estimate, $K($ hits $) /[1-R$ (hits) $] .{ }^{* *} p<.005$. 
Table 3

Correlations Between Description Quality and Accuracy Measures for Distinctive Faces

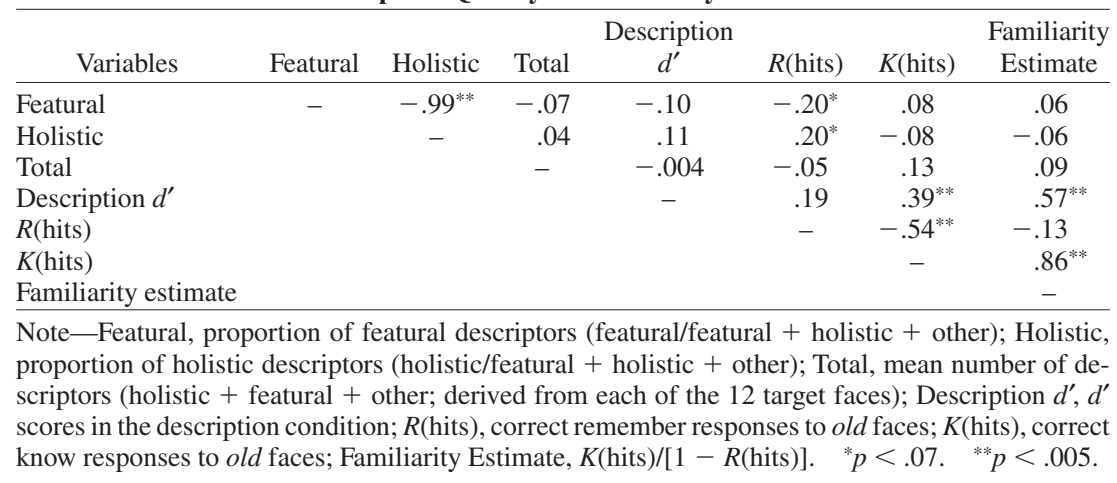

descriptors, know responses must be negatively correlated with featural descriptors.) There was no correlation between description quality and familiarity estimates.

For distinctive faces, a similar pattern emerged. There was a trend toward a decrease in the proportion of featural descriptors and an increase in the proportion of holistic descriptors being associated with an increase in correct remember responses.

\section{DISCUSSION}

In a novel paradigm, we have established that verbally describing a visual memory of a face can benefit subsequent visual recognition of that same face. There were two additional findings. First, verbalization benefited face recognition independently of visual distinctiveness; verbal facilitation was equally strong for both typical and distinctive faces (and a careful analysis of description quality showed no difference between faces allotted to these two categories). Second, both verbalization and visual distinctiveness enhanced recollection, but their effects on familiarity-based recognition were less clear-cut. We will discuss each of these findings in turn.

\section{Verbalization and Visual Distinctiveness}

The facilitative effects of verbalization observed here contrast with the effects of verbal interference that have been documented elsewhere (e.g., Brown \& Lloyd-Jones, 2002, 2003; Meissner et al., 2001; Schooler \& EngstlerSchooler, 1990). In particular, Brown and Lloyd-Jones $(2002,2003)$ found verbal interference in a paradigm in which participants described a single face following the presentation of a series of different faces. They argued that verbal interference is mediated by a generalized shift in processing style from more holistic visual processing to the processing of individual facial features, which are less useful for subsequent recognition. However, in the present paradigm, the participants described each face in the series. Thus, we argue that the facilitative effects of verbalization arise from an alteration to the memory representation of a particular face, rather than from some generalized strategy. In agreement with this proposal, a relationship was found between the quality of the partici- pants' descriptions and their recollection-based recognition memory. Generating fewer featural descriptors and more holistic descriptors was associated with an increase in recollection. Nevertheless, there are also other differences between the present paradigm and more traditional paradigms with which verbal overshadowing has been obtained. These include (1) the amount of time given to describing the stimulus, (2) the nature of the control task, and (3) the form of assessment used (e.g., old/new recognition vs. identification with a lineup procedure). Such differences may prove to be important in observing the facilitative and interfering effects of verbalization on face memory.

In principle, verbal facilitation may be mediated by an alteration to either a visual or a semantic memory representation of a particular face. However, the effects of verbalization were not modulated by visual distinctiveness, and therefore, the present findings do not support a visualprocessing account in which facilitation arises as a function of the quantity (e.g., Winograd, 1978) or quality (e.g., Wells \& Hryciw, 1984) of visual information encoded from the face. Rather, the findings are more consistent with a semantic-processing account, whereby facilitation allows for the formation of richer semantic associations with the described face, which benefits retrieval (e.g., Anderson \& Reder, 1979; Bruce \& Young, 1986; Ryan \& Schooler, 1994, cited in Schooler et al., 1996).

We should note, however, that the present paradigm differs from previous paradigms with which the effects of verbal facilitation on face recognition have been investigated, since the participants described their visual memory of a face, rather than making a judgment about the face while it was present. It is possible, therefore, that processes other than those outlined above may have come into play. For instance, verbalization may provide an action tag to the stored visual memory of an unfamiliar face that helps to differentiate it from other faces that have been presented. An action tag may be thought of as additional information that is attached to the visual memory representation and specifies visual or semantic aspects of the face that are useful (or diagnostic) for successful recognition. When the face is re-presented, the action tag specifies, or directs processing toward, information relevant to the task (see 
DeSchepper \& Treisman, 1996; Neill \& Valdes, 1992; Ryan \& Schooler, 1994, cited in Schooler et al., 1996).

The paradigm developed here, which allows for both free descriptions and descriptions in which participants are constrained to describe faces in a particular way and which allows the association of qualitative and quantitative aspects of face descriptors with recognition performance, should provide further insight into the nature of the effects of verbalization on face recognition.

\section{Recollection and Familiarity}

Verbalization enhanced face recollection, as distinct from familiarity. This is consistent with a broad range of studies showing that recollection benefits (and generally more so than familiarity) from elaborative processing, such as that with meaningful, as compared with perceptual, processing or with active generation, as compared with passive processing (for reviews, see Rugg \& Yonelinas, 2003; Yonelinas, 2002). Visual distinctiveness also independently enhanced recollection. This, too, is consistent with a number of previous studies-for instance, those in which words, pictures, and faces were used as stimuli (e.g., Brandt et al., 2003; Dewhurst \& Conway, 1994; Mäntylä, 1997).

The findings concerning familiarity-based recognition were mixed. Neither verbalization nor visual distinctiveness influenced (correct) know responses. However, when the independence remember/know procedure of Yonelinas (2002) was applied, both verbalization and visual distinctiveness independently enhanced familiarity. Previous studies have not (to our knowledge) examined the effects of verbalization on familiarity. However, in two recent studies, the effects of visual distinctiveness on familiarity, as assessed through know responses, have been examined.

First, in the only other study to have examined the effects of visual distinctiveness on remember and know responses in face recognition, Brandt et al. (2003, Experiment 1) found that typical faces elicited more know responses than did distinctive faces. However, two caveats should be noted: (1) The finding was not replicated in a subsequent experiment, and (2) when the accuracy of know responses is measured taking into account false alarm rates, there is no longer an effect of visual distinctiveness (i.e., when subtracting false alarms from hits; cf. Kishiyama \& Yonelinas, 2003). Second, a study by Kishiyama and Yonelinas showed that distinctiveness of object images (created by presenting them in an arbitrarily different color) did not benefit know responses. However, as is the case here, distinctiveness was seen to benefit an estimate of familiarity derived from the independence remember/know procedure.

How might we account for the fact that verbalization and visual distinctiveness influence measures that, according to some dual-process models, are assumed to tap functionally (and perhaps, also neurally) distinct memorial processes of recollection and familiarity? Two possibilities come from Kishiyama and Yonelinas (2003).
First, visual distinctiveness may influence recollection through elaborative encoding operations and may influence familiarity-based recognition by reducing interference at retrieval. In the present study, we found that visual distinctiveness influenced know responses to new faces. More specifically, distinctive faces elicited fewer false know responses than did typical faces. This is consistent with an influence on retrieval. Note, also, that we found similar effects for verbalization. Second, there may be both automatic and controlled responses during encoding, and these two responses may influence familiarity and recollection, respectively. Thus verbalized and distinctive items may lead to an automatic orienting response that may enhance subsequent familiarity-based recognition, and in addition, verbalization and distinctiveness may also lead to further elaborative or controlled processing (see also Schmidt, 1991).

Nevertheless, on both accounts, one would expect to observe a dissociation between remember and know responses with the independence remember/know procedure, and that was not the case here. Effects of verbalization and visual distinctiveness were evident for both remember and know responses (for instance, there was a description - no description difference of .09 vs. .07 for recollection and familiarity estimates, respectively).

In fact, the present findings are more consistent with accounts that do not always predict a dissociation between remember and know judgments. One such (singleprocess) account has been proposed by Donaldson (1996), who argued that recollection is assumed to reflect the retrieval of strong content-rich memories, whereas familiarity is associated with weaker, less specific memories. Alternatively, several authors have proposed dual-process accounts whereby remember and know judgments are not characterized as process-pure measures of recollection and familiarity. Rather, these judgments reflect the combined contributions of recollection and familiarity processes (see, e.g., Banks, 2000; Rotello, Macmillan, \& Reder, 2004; Wixted \& Stretch, 2004). For instance, Rotello et al. suggested that remember and know responses reflect the contributions of two phenomenologically distinct forms of memory: global familiarity and specific recollection. However, memory strength varies as a function of both global, overall strength and the specific strength of details associated with test items. Thus, global memory strength can be correlated with or contradicted by the specific strength of particular details. From this perspective, remember and know judgments may not be particularly well suited to estimating the contributions of the familiarity and recollective processes to recognition. Rather, theoretical development will require the development of mathematical models.

The present study has provided the first demonstration that both recollection- and familiarity-based face recognition processes can be influenced by verbalization. Although there is substantial evidence that recollection and familiarity can be dissociated through use of a number of different variables, the findings presented here sug- 
gest that neither verbalization nor visual distinctiveness is particularly suited to differentiating between these two processes in the remember/know paradigm.

\section{REFERENCES}

Anderson, J. R., \& REDER, L. M. (1979). An elaborative processing explanation of depth of processing. In L. S. Cermak \& F. I. M. Craik (Eds.), Levels of processing in human memory (pp. 385-395). Hillsdale, NJ: Erlbaum.

Baddeley, A. D., \& Woodhead, M. (1983). Improving face recognition ability. In S. M. A. Lloyd-Bostock \& B. R. Clifford (Eds.), Evaluating witness evidence (pp. 125-136). Chichester, U.K.: Wiley.

BANKs, W. P. (2000). Recognition and source memory as multivariate decision processes. Psychological Science, 11, 267-273.

Benson, P. J., \& Perrett, D. I. (1994). Visual processing of facial distinctiveness. Perception, 23, 75-93.

Berman, G. L., \& Cutler, B. L. (1998). The influence of processing instructions at encoding and retrieval on face recognition accuracy. Psychology, Crime \& Law, 4, 89-106.

Blaney, R. L., \& Winograd, E. (1978). Developmental differences in children's recognition memory for faces. Developmental Psychology, 14, 441-442.

Bloom, L., \& Mudd, S. A. (1991). Depth of processing approach to face recognition: A test of two theories. Journal of Experimental Psychology: Learning, Memory, \& Cognition, 17, 556-565.

Bower, G. H., \& Karlin, M. B. (1974). Depth of processing pictures of faces and recognition memory. Journal of Experimental Psychology, 103, $751-757$.

Brandt, K. R., Macrae, C. N., Schloerscheidt, A. M., \& Milne, A. B. (2003). Remembering or knowing others? Person recognition and recollective experience. Memory, 11, 89-100.

Brown, C., \& Lloyd-Jones, T. J. (2002). Verbal overshadowing in a multiple face presentation paradigm: Effects of description instruction. Applied Cognitive Psychology, 16, 873-885.

Brown, C., \& Lloyd-Jones, T. J. (2003). Verbal overshadowing of multiple face and car recognition: Effects of within- versus acrosscategory verbal descriptions. Applied Cognitive Psychology, 17, 183 201.

Bruce, V., Burton, A. M., \& Dench, N. (1994). What's distinctive about a distinctive face? Quarterly Journal of Experimental Psychology, 47A, 119-141.

Bruce, V., \& Young, A. W. (1986). Understanding face recognition. British Journal of Psychology, 77, 305-327.

Burton, A. M., Bruce, V., \& Hancock, P. J. B. (1999). From pixels to people: A model of familiar face recognition. Cognitive Science, 23, 1-31.

Coin, C., \& Tiberghien, G. (1997). Encoding activity and face recognition. Memory, 5, 545-568.

Courtois, M. R., \& Mueller, J. H. (1979). Processing multiple physical features in facial recognition. Bulletin of the Psychonomic Society, 14, 74-76.

Craik, F. I. M., \& Lockhart, R. S. (1972). Levels of processing: A framework for memory research. Journal of Verbal Learning \& Verbal Behavior, 11, 671-684.

Davies, G., Shepherd, J., \& Ellis, H. (1979). Effects of interpolated mugshot exposure on accuracy of eyewitness identification. Journal of Applied Psychology, 64, 232-237.

Deffenbacher, K. A., Carr, T. H., \& Leu, J. R. (1981). Memory for words, pictures, and faces: Retroactive interference, forgetting and reminiscence. Journal of Experimental Psychology: Human Learning \& Memory, 7, 299-305.

DeSchepper, B., \& Treisman, A. (1996). Visual memory for novel shapes: Implicit coding without attention. Journal of Experimental Psychology: Learning, Memory, \& Cognition, 22, 27-47.

Dewhurst, S. A., \& Conway, M. A. (1994). Pictures, images, and recollective experience. Journal of Experimental Psychology: Learning, Memory, \& Cognition, 20, 1088-1098.
Dodson, C. S., Johnson, M. K., \& Schooler, J. W. (1997). The verbal overshadowing effect: Why descriptions impair facial recognition. Memory \& Cognition, 25, 129-139.

DonAlDSON, W. (1996). The role of decision processes in remembering and knowing. Memory \& Cognition, 24, 523-533.

Ellis, H. D., Shepherd, J. W., \& Davies, G. M. (1980). The deterioration of verbal descriptions of faces over different delay intervals. Journal of Police Science \& Administration, 8, 101-106.

GARDINER, J. M. (1988). Functional aspects of recollective experience. Memory \& Cognition, 16, 309-313.

GARDINER, J. M., \& JAVA, R. I. (1990). Recollective experience in word and nonword recognition. Memory \& Cognition, 18, 23-30.

Gardiner, J. M., \& PARKIN, A. J. (1990). Attention and recollective experience in recognition memory. Memory \& Cognition, 18, 579-583.

Hancock, P. J. B., Burton, A. M., \& Bruce, V. (1996). Face processing: Human perception and principal components analysis. Memory \& Cognition, 24, 26-40.

KerR, N. H., \& WinOGRAD, E. (1982). Effects of contextual elaboration on face recognition. Memory \& Cognition, 10, 603-609.

Kishiyama, M. M., \& Yonelinas, A. P. (2003). Novelty effects on recollection and familiarity in recognition memory. Memory \& Cognition, 31, 1045-1051.

Klatzky, R. L., Martin, G. L., \& Kane, R. A. (1982). Semantic interpretation effects on memory for faces. Memory \& Cognition, 10, 195-206.

Leder, H., \& Bruce, V. (1998). Local and relational aspects of face distinctiveness. Quarterly Journal of Experimental Psychology, 51A, 449-473.

LEWIS, M. B. (2004). Face-space-R: Towards a unified account of face recognition. Visual Cognition, 11, 29-69.

Light, L. L., Kayra-Stuart, F., \& Hollander, S. (1979). Recognition memory for typical and unusual faces. Journal of Experimental Psychology: Human Learning \& Memory, 5, 212-228.

Lindsay, R. C. L., Martin, R., \& Webber, L. (1994). Default values in eyewitness descriptions: A problem for the match-to-description lineup foil selection strategy. Law \& Human Behavior, 18, 527-541.

MÄNTYLÄ, T. (1997). Recollections of faces: Remembering differences and knowing similarities. Journal of Experimental Psychology: Learning, Memory, \& Cognition, 23, 1203-1216.

Meissner, C. A., \& Brigham, J. C. (2001). A meta-analysis of the verbal overshadowing effect in face identification. Applied Cognitive Psychology, 15, 603-616.

Meissner, C. A., Brigham, J. C., \& Kelley, C. M. (2001). The influence of retrieval processes in verbal overshadowing. Memory \& Cognition, 29, 176-186.

Mueller, J. H., Courtois, M. R., \& Bailis, K. L. (1981). Self-reference in facial recognition. Bulletin of the Psychonomic Society, 17, 85-88.

NeILl, W. T., \& VAldes, L. A. (1992). Persistence of negative priming: Steady state or decay? Journal of Experimental Psychology: Learning, Memory, \& Cognition, 18, 565-576.

O’Toole, A. J., Deffenbacher, K. A., Valentin, D., \& Abdi, H. (1994). Structural aspects of face recognition and the other-race effect. Memory \& Cognition, 22, 208-224.

Parkin, A. J., Gardiner, J. M., \& Rosser, R. (1995). Functional aspects of recollective experience in face recognition. Consciousness \& Cognition, 4, 387-398.

PAtTerson, K. E., \& BAdDeley, A. D. (1977). When face recognition fails. Journal of Experimental Psychology: Human Learning \& Memory, 3, 406-417.

Richardson-Klavehn, A., Gardiner, J. M., \& JaVA, R. I. (1996). Memory: Task dissociations, process dissociations and dissociations of consciousness. In G. D. M. Underwood (Ed.), Implicit cognition (pp. 85-158). Oxford: Oxford University Press.

Rotello, C. M., Macmillan, N. A., \& Reder, J. A. (2004). Sumdifference theory of remembering and knowing: A two-dimensional signal-detection model. Psychological Review, 111, 588-616.

RugG, M. D., \& Yonelinas, A. P. (2003). Human recognition memory: A cognitive neuroscience perspective. Trends in Cognitive Sciences, 7, 313-319. 
Schmidt, S. R. (1991). Can we have a distinctive theory of memory? Memory \& Cognition, 19, 523-542.

Schooler, J. W. (2002). Verbalization produces a transfer inappropriate processing shift. Applied Cognitive Psychology, 16, 989-997.

Schooler, J. W., \& Engstler-Schooler, T. Y. (1990). Verbal overshadowing of visual memories: Some things are better left unsaid. Cognitive Psychology, 22, 36-71.

Schooler, J. W., Fiore, S. M., \& Brandimonte, M. A. (1997). At a loss from words: Verbal overshadowing of perceptual memories. In D. L. Medin (Ed.), The psychology of learning and motivation: Advances in research and theory (Vol. 37, pp. 291-340). San Diego: Academic Press.

Schooler, J. W., Ryan, R. S., \& Reder, L. (1996). The costs and benefits of verbally rehearsing memory for faces. In D. J. Herrmann, C. McEvoy, C. Hertzog, P. Hertel, \& M. K. Johnson (Eds.), Basic and applied memory research: Vol. 2. Practical applications (pp. 51-65). Mahwah, NJ: Erlbaum.

Shapiro, P. N., \& Penrod, S. (1986). Meta-analysis of facial identification studies. Psychological Bulletin, 100, 139-156.

Snodgrass, J. G., \& Corwin, J. (1988). Pragmatics of measuring recognition memory: Applications to dementia and amnesia. Journal of Experimental Psychology: General, 117, 34-50.

Sporer, S. L. (1991). Deep-deeper-deepest? Encoding strategies and the recognition of human faces. Journal of Experimental Psychology: Learning, Memory, \& Cognition, 17, 323-333.

Tulving, E. (1985). Memory and consciousness. Canadian Journal of Psychology, 26, 1-12.

VAlentine, T. (1991). A unified account of the effects of distinctiveness, inversion, and race in face recognition. Quarterly Journal of Experimental Psychology, 43A, 161-204.
Valentine, T., \& Bruce, V. (1986). The effects of distinctiveness in recognising and classifying faces. Perception, 15, 525-535.

VAlentine, T., \& Endo, M. (1992). Towards an exemplar model of face processing: The effects of race and distinctiveness. Quarterly Journal of Experimental Psychology, 44A, 671-703.

Wells, G. L., \& Hryciw, B. (1984). Memory for faces: Encoding and retrieval operations. Memory \& Cognition, 12, 338-344.

Westerman, D. L., \& Larsen, J. D. (1997). Verbal-overshadowing effect: Evidence for a general shift in processing. American Journal of Psychology, 110, 417-428.

WiNOGRAD, E. (1978). Encoding operations which facilitate memory across the lifespan. In M. M. Gruneberg, P. E. Morris, \& R. N. Sykes (Eds.), Practical aspects of memory (pp. 252-262). San Diego: Academic Press.

WinOGRAD, E. (1981). Elaboration and distinctiveness in memory for faces. Journal of Experimental Psychology: Human Learning \& Memory, 7, 181-190.

Wixted, J. T., \& Stretch, V. (2004). In defense of the signal detection interpretation of remember/know judgments. Psychonomic Bulletin \& Review, 11, 616-641.

Yonelinas, A. P. (2002). The nature of recollection and familiarity: A review of 30 years of research. Journal of Memory \& Language, 46, 441-517.

Yonelinas, A. P., \& JACOBY, L. L. (1995). The relation between remembering and knowing as bases for recognition: Effects of size congruency. Journal of Memory \& Language, 34, 622-643.

(Manuscript received October 8, 2004; revision accepted for publication March 17, 2005.) 